\title{
Single-Cell Analysis: from Innovative Omics to Target Therapy
}

\section{Ferdinando Mannello*}

Department of Biomolecular Sciences, Section of Clinical Biochemistry, Unit of Cell Biology, University "Carlo Bo" of Urbino, Via O. Ubaldini 7, 61029 Urbino (PU), Italy

To date, most of cell biology studies were performed on cell clusters, considering that all cells belonging to a peculiar cell type were identical. The renewed interest in cell-to-cell variation and its characterization enriched the concept of cellular heterogeneity, so far use, especially referred to cancer phenotypization [1].

Thanks to the significant technological improvements, actually linked to the miniaturization of device (that give rise to the "Lab-OnChip Era") and to an improved detection sensitivity of methods, an increasingly number of studies on single cells shows that individual cells within the same isogenic population may differ dramatically, and these differences drive important consequences for the health and cellular function of the entire population [2].

Methodological approaches evaluating only parameters, resulting from the averages of large population of cells, fail to detect and consider cells produced in small number, and attempt to uniform the heterogeneity, obscuring crucial differences. On the contrary, the development of new technologies to investigate biological systems at single-cell level is characterized by enhanced measurement parameters (selectivity, sensitivity, spatiotemporal resolution, scalability, and/ or non-destructive methods that preserve the integrity of the cell, allowing the analyzing of intracellular bio-molecular dynamics). These new tools are also able to perform a simultaneous measurement of multiple molecular components (like genes, proteins and metabolites), within a single cell [3].

For these reasons, genomics, proteomics and metabolomics experimental approaches shifted at single-cell level could be considered of primary importance to define the normal cell-to-cell variation, and correlate the variation with changes in biological function and disease processes, to uncover crucial intra- and extra-cellular pathways (also, in response to microenvironmental stimuli) related to a peculiar genome polymorphism, to improve the detection and molecular characterization of rare cells (e.g. stem cells, cancer stem cells, and tumor initiating cells), and finally to identify personalized pharmacological treatments.

Moreover in the last years, there was an increasing interest in investigating cellular heterogeneity involved in different fields, from cancer to stem cell research, from autoimmune disease to neuronal biology, through the outstanding use of single-cell analyses with omic approaches, resulting in a more efficient discrimination among responders and non-responders to several therapeutic approaches.

The introduction of modern genomics increased the number of whole genome expression profiles, discovered in different organisms (bacteria, plants, humans and others), with the final goal to determine the exact type and number of genes and proteins expressed in a different tissue or organ, the expression level and regulative network of these genes and proteins, both in physiological and in pathological conditions.

Nevertheless, only with the advent of microfluidic systems and miniaturized devices, nearer to the effective dimension of cells and suitable to perform analyses even in very small volume sample, it has been possible to discern different gene expression and protein phenotype, in order to distinguish crucial associated functions in cell populations that seem "apparently" anatomically and morphologically identical [4].

At this regard, it has been recently demonstrated for the first time, the possibility to distinguish the pluripotential and differentiated state of among seemingly homogenous cell populations, by resolving stem cell heterogeneity with single-cell gene expression profiles. This discovery is relevant particularly, when we think about the applicability of stem cell in regenerative medicine, as an intervention strategy enabling stem cells to get in the damaged tissue and repopulate/ differentiate in a tissue-committed cell-type [5]. Nevertheless, it has been yet to be clarified the exact mechanism by which stem cells interact with diseased tissues, and how microenvironments influence their activity [6].

In the field of cancer research, the exact detection and evaluation of the single cell composing the heterogeneous mass set the basis for the comprehension of the mechanisms underlying the initiation, progression and metastatization of different type of cancer, such as for breast cancer [7].

At this regard, it is well known that molecular heterogeneity of breast cancer [8], is mainly referred to the different cell-type composition of the tumor and of the mammary microenvironment (breast epithelial stem cells, transit amplifying cells, committed differentiated cells) [9]. The further and more relevant difference in cell composition of human cancer is the different genomic, transcriptomic, epigenomic, proteomic and metabolomic profiles within the apparently identical single cell type, in order to not only clarify the different steps involved in breast cancer evolution, but also to understand drug therapy resistance and cancer reoccurrence $[10,11]$.

Regarding the cancer stem routes, it has been recently emerged as an international stem-cell debate underlying the two current thoughts on the possible origin of cancer cells ("clonal tumour evolution" versus "stemming tumour evolution") [12], high lightening that single-cell approaches may pave the way for a complete and innovative knowledge of molecular portraits.

Furthermore, not only the cell-type is the one that gives rise to the tumour mass (like cancer stem cells), but also the circulating tumour

*Corresponding author: Ferdinando Mannello, Department of Biomolecular Sciences, Section of Clinical Biochemistry, Unit of Cell Biology, University "Carlo Bo" of Urbino, Via O. Ubaldini 7, 61029 Urbino (PU), Italy, Tel: +39 0722 351479; Fax: +39 0722 322370; E-mail: ferdinando.mannello@uniurb.it

Received November 23, 2012; Accepted November 24, 2012; Published November 27, 2012

Citation: Mannello F (2012) Single-Cell Analysis: from Innovative Omics to Target Therapy. J Pharmacogenom Pharmacoproteomics 3: e130. doi:10.4172/21530645.1000e130

Copyright: () 2012 Mannello F. This is an open-access article distributed under the terms of the Creative Commons Attribution License, which permits unrestricted use, distribution, and reproduction in any medium, provided the original author and source are credited. 
cells may play a crucial role in the progression of cancer and in the drug therapy response; so their detection at single-cell level may represent a powerful tool to predict an increased risk for disease relapse and establish the correct prognosis. In this respect, the analyses of different single-cell omics could find crucial involvement [13].

The indispensable step after the isolation of a single cancer cell (stem, not stem, circulating or resident cells), and after its wholegenome, whole-transcriptome and whole-proteome characterization is the study of a pharmacological approach targeted to effectively treat the cancer cell, by killing or reprogramming the pathological feature(s). This preliminary condition is necessary, but not sufficient to develop pharmacogenomic and pharmacoproteomic strategies for a more efficient personalized medicine, as it must deal with the reproducibility of the response to treatment, and both with the effects induced in the other cancer and no-cancer cells, and with the entire organism networks.

Only with an adequate evaluation of the complexity of cancer genome and with an advanced and multidisciplinary study of single cancer cell as unique and heterogeneous entity, pharmacogenomics could be translated into clinic, in order to improve management of patients $[14,15]$.

Another goal useful to translate genomic data (to date characterized by extremely large databases of disease-associated mutations) in new pharmacogenomic-based therapeutics, is also the comprehension of which mutations/alterations in pathological cells are "driving the diseased condition" [16]

Furthermore, the single-cell analysis has been also applied to neuroscience, in order to understand if the genomic heterogeneity among single-neuron cells is involved in their functional diversity, and how this contributes to different neurological and psychiatric disorders [17].

Also, single-cell transcriptome has been employed to characterize brain cell-to-cell variations in order to reveal, through the studies of gene splicing isoforms, novel regulatory mechanisms underlying the phenotypic differences between genetically identical cells $[18,19]$.

As a complement of genomic analyses, recent proteomic studies at single-cell level, shedding lights on protein structure, function, subcellular localization, networks and modification in physiopathiological conditions [20]. This offer a powerful tool for discovery of innovative and more effective drugs that could be used for individualized therapy, both in the treatment of cancer [7], also by studying single-rare cell response to treatment [21], brain disorders [22], immune cells and related disorders [23].

The increasing number of cell biology studies at the single-cell level promoted the discovery of the existence of millions of different cell types, both in the prokaryotic and in the eukaryotic spheres. The conventional morphological and functional characterization of cells is now integrated by modern-omics and imaging technologies, in order to transform medical research and clinical practice in an unprecedented rate [24]. Thanks to these technological and scientific advancements, it will be possible to reveal previously undetectable differences in the biomolecular composition of individual cells, in order to set the basis for detailed health informations ad personam (e.g. whole-genome, wholetranscriptome and whole-proteome profile), that would be usefully employed in clinic, both to perform a more exact prediction of disease susceptibility and diagnosis, and to finally develop a personalized therapeutic strategy [25].

Even if a great amount of progress in this field has been done in order to translate experimental single-cell pharmacogenomic studies into clinical practice, there is the urgent need of further improvements (e.g. innovative high resolution cell imaging methods, platform measuring simultaneously chemical, physical, molecular, structural and electrical cell properties among individual cells, etc.), in order to transform the "old" knowledge of systems biology through the "new" insights derived from cellular heterogeneity by single-cell studies.

\section{References}

1. Mannello F, Ligi D (2013) Resolving the cell heterogeneity of tumours and searching reliable protein cancer biomarkers in breast microenvironment. BMC Cancer (in press).

2. Mannello F, Ligi D, Magnani M (2012) Deciphering the single-cell-Omic: Innovative application for translational medicine. Expert Rev Proteomic (in press).

3. Lecault V, White AK, Singhal A, Hansen CL (2012) Microfluidic single cel analysis: from promise to practice. Curr Opin Chem Biol 16: 381-390.

4. Kawasaki ES (2004) Microarray and gene expression profile of a single cell. Ann N Y Acad Sci 1020: 92-100.

5. Narsinh KH, Sun N, Sanchez-Freire V, Lee AS, Almeida P, et al. (2011) Single cell transcriptional profiling reveals heterogeneity of human induced pluripotent stem cells. J Clin Invest 121: 1217-1221.

6. Ho JWS (2012) Mechanisms of Action of Proteins, Peptides and Biomarkers in Single Cell Analysis. Single Cell Biol 1: e115

7. Shi Q, Qin L, Wei W, Geng F, Fan R, et al. (2012) Single-cell proteomic chip for profiling intracellular signaling pathways in single tumor cells. Proc Natl Acad Sci U S A 109: 419-424.

8. Shipitsin M, Campbell LL, Argani P, Weremowicz S, Bloushtain-Qimron N, et al. (2007) Molecular definition of breast tumor heterogeneity. Cancer Cell 11 259-273.

9. Stingl J, Caldas C (2007) Molecular heterogeneity of breast carcinomas and the cancer stem cell hypothesis. Nat Rev Cancer 7: 791-799.

10. Ng CK, Pemberton HN, Reis-Filho JS (2012) Breast cancer intratumor genetic heterogeneity: causes and implications. Expert Rev Anticancer Ther 12: 1021 1032.

11. Heselmeyer-Haddad K, Berroa Garcia LY, Bradley A, Ortiz-Melendez C, Lee WJ, et al. (2012) Single-Cell Genetic Analysis of Ductal Carcinoma in Situ and Invasive Breast Cancer Reveals Enormous Tumor Heterogeneity yet Conserved Genomic Imbalances and Gain of MYC during Progression. Am J Pathol 181: 1807-1822

12. Gilbertson RJ, Graham TA (2012) Cancer: Resolving the stem-cell debate Nature 488: 462-463.

13. Powell AA, Talasaz AH, Zhang H, Coram MA, Reddy A, et al. (2012) Single cell profiling of circulating tumor cells: transcriptional heterogeneity and diversity from breast cancer cell lines. PLoS One 7: e33788.

14. Samuel N, Hudson TJ (2012) Translating Genomics to the Clinic: Implications of Cancer Heterogeneity. Clin Chem.

15. Hartmaier RJ, Priedigkeit N, Lee AV (2012) Who's driving anyway? Herculean efforts to identify the drivers of breast cancer. Breast Cancer Res 14: 323.

16. Ha BH, Boggon TJ (2012) Understanding the Molecular and Functional Mechanisms that Underlie Pharamcogenomics-Based Therapy. J Pharmacogenom Pharmacoproteomics 3:4.

17. Evrony GD, Cai X, Lee E, Hills LB, Elhosary PC, et al. (2012) Single-neuron sequencing analysis of $I 1$ retrotransposition and somatic mutation in the human brain. Cell 151: 483-496.

18. Vidaurre OG, Gascón S, Deogracias R, Sobrado M, Cuadrado E, et al 
Citation: Mannello F (2012) Single-Cell Analysis: from Innovative Omics to Target Therapy. J Pharmacogenom Pharmacoproteomics 3: e130. doi:10.4172/2153-0645.1000e130

Page 3 of 3

(2012) Imbalance of neurotrophin receptor isoforms TrkB-FL/TrkB-T1 induces neuronal death in excitotoxicity. Cell Death Dis 3: e256.

19. Eberwine J, Yeh H, Miyashiro K, Cao Y, Nair S, et al. (1992) Analysis of gene expression in single live neurons. Proc Natl Acad Sci U S A 89: 3010-3014.

20. Wu M, Singh AK (2012) Single-cell protein analysis. Curr Opin Biotechnol 23: 83-88.

21. Faley SL, Copland M, Reboud J, Cooper JM (2011) Cell chip array for microfluidic proteomics enabling rapid in situ assessment of intracellular protein phosphorylation. Biomicrofluidics 5: 24106.

22. Rubakhin SS, Sweedler JV (2008) Quantitative measurements of cell-cell signaling peptides with single-cell MALDI MS. Anal Chem 80: 7128-7136.

23. Kim SM, Bhonsle L, Besgen P, Nickel J, Backes A, et al. (2012) Analysis of the paired TCR $\alpha$ - and $\beta$-chains of single human T cells. PLoS One 7: e37338.

24. Eberwine J, Lovatt D, Buckley P, Dueck H, Francis C, et al. (2012) Quantitative biology of single neurons. J R Soc Interface 9: 3165-3183.

25. Hood L, Tian Q (2012) Systems approaches to biology and disease enable translational systems medicine. Genomics Proteomics Bioinformatics 10: 181 185 